\title{
A real-time criterion theory of duration discrimination
}

\author{
ALFRED B. KRISTOFFERSON \\ McMaster University, Hamilton, Ontario, Canada
}

\begin{abstract}
The common idea that a measure is taken of a duration stimulus over its temporal extent, and that the decision as to whether the stimulus is relatively long or short is based upon such a measure, is shown to be incorrect. Two experiments, which require speeded responding in duration discrimination and consider response latencies as well as response probabilities, demonstrate that the response that is made is determined by the outcome of a race between an internally timed interval, the criterion, and the presented stimulus. The onset of the stimulus triggers the criterion; if the criterion ends first, the response "long" is elicited. Duration discrimination is a matter of temporal order discrimination, and in the limit, "short" responses are simple reactions while "long" responses are time estimation responses. A specific model of the real-time criterion hypothesis is tested, and these initial tests generally confirm it. From this, it is concluded that errors in duration discrimination are due entirely to variability of the criterion and that afferent latencies are not necessarily variable. This adds additional evidence for the existence of deterministic afferent latencies.
\end{abstract}

Previous theories of duration discrimination have assumed as an input process some mechanism which takes a measure of the temporal extent of the duration stimulus (see the review by Allan \& Kristofferson, 1974a). Such an interval-measure hypothesis is not the only possibility, as the review also pointed out, and one purpose of the present paper is to demonstrate that that hypothesis is incorrect, at least under certain conditions which often prevail in duration discrimination experiments. ${ }^{1}$

In many experimental situations, the metric information about the presented stimulus that is made available by the interval-measure hypothesis is more than is necessary to make the discrimination. In such situations, mechanisms which provide only order information are sufficient. A second purpose of this paper is to provide a specific mechanism of this kind. It is one of a class of mechanisms which follow from a real-time criterion hypothesis of the input process, a hypothesis which is an alternative to that of interval measure.

The real-time criterion hypothesis is shown in Figure 1 and will be discussed in the context of a particular discrimination situation which uses the many-to-few method (Allan \& Kristofferson, 1974b). A set of four duration stimuli is shown at the top of Figure 1. Each stimulus consists, say, of two identical 10 -msec auditory pulses, $P_{1}$ and $P_{2}$, which occur in succession. The onset of $P_{2}$ occurs $D$ msec after

This research was supported by Grant A7919 from the National Research Council of Canada. Much of the paper was written while the author was on leave at the MRC-Applied Psychology Unit, Cambridge, England. I thank Michael Bishop for his excellent technical assistance. the onset of $P_{1}$, and the stimuli differ only in D. On each trial, one stimulus only is presented and one of two responses is required. A response meaning short, $R(S)$, is correct for $S_{1}$ and $S_{2}$, and the second response, $R(L)$, is correct for $S_{3}$ and $S_{4}$. This application of the method divides the set of values of $D$ at the midpoint of the set, and that midpoint (MP) is the base duration. The proportion of responses which are $R(L)$, called $P(L)$, for each $S$, are the discrimination data.

The real-time criterion hypothesis is shown at the bottom of Figure 1. It states that a criterion, $\mathrm{C}$,
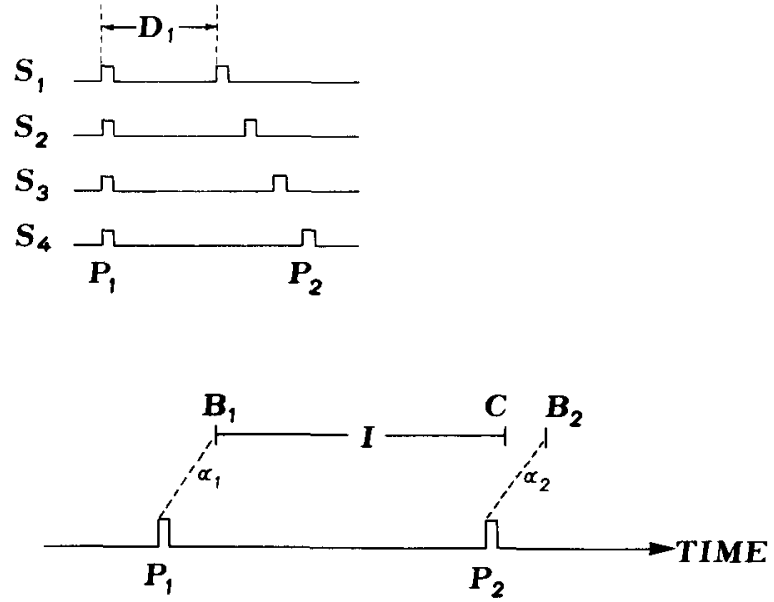

Figure 1. Above: A set of four duration stimuli. The stimulus duration of $S_{1}$ is $D_{1}$, the onset-to-onset time of the two brief energy pulses. Below: Schematic of the real-time criterion hypothesis. Each energy pulse causes an internal event $B$ following afferent latency $\alpha$. Another internal event $C$, is triggered by $B_{1}$ after an internally timed delay $I$. The response $R(L)$ occurs if $C$ precedes $B_{2}$, and $R(S)$ occurs whenever $B_{2}$ precedes $C$. 
which is an internal time-point event triggered by $P_{1}$, occurs following $P_{1}$ by a time which is the sum of $\alpha_{1}$, an afferent latency of $P_{1}$, and $I$, which is an internally timed interval. A second internal timepoint event $B_{2}$ is caused by $P_{2}$ and occurs following an afferent latency of $\alpha_{2}$. Response choice is determined by the temporal relationship between $C$ and $B_{2}$. A response $R(L)$ results when $C$ occurs first and $R(S)$ when $B_{2}$ occurs first. In this view, duration discrimination is seen to be a matter of temporal order discrimination, and the goal of integrating these phenomena, along with successiveness discrimination, seems nearer (Allan, 1975; Kristofferson \& Allan, 1973).

The time at which $\mathrm{C}$ occurs can be adjusted by changing the sum of $\alpha_{1}$ and I. In the single-stimulus procedure under discussion here, this adjustment would be made in the course of practice with the stimulus set, and $C$ should come to be placed near (MP $\left.+\alpha_{2}\right)$. With two-alternative forced choice, it is possible that the first stimulus sets a value of $\left(\alpha_{1}+\mathrm{I}\right)$, which is then used to evaluate the second stimulus. ${ }^{2}$

There is a clear basis for choosing between the two general hypotheses about the input process. The real-time criterion hypothesis says that $R(L)$ is triggered by $C$, which in turn was triggered by $P_{1}$. The other response, $R(S)$, is triggered by $P_{2}$ by way of $B_{2}$. Thus, there are two distinct causal chains: $P_{1}-B_{1}-C-R(L)$ and $P_{2}-B_{2}-R(S)$. In general, $R(S)$ should be time-locked to $P_{2}$ and $R(L)$ to $P_{1}$. Short responses should always occur after $\mathbf{P}_{2}$ (i.e., after the end of the stimulus duration), and the time following $P_{1}$ at which $R(S)$ occurs should increase with increases in $D$. But the time at which $R(L)$ occurs should be independent of the end of the stimulus duration and, in general but with an exception to be explained later, $R(L)$ should occur at the same time with respect to $P_{1}$ regardless of the duration of the stimulus. In fact, it is quite possible for $R(L)$ to be made correctly even before the stimulus duration has ended, a happening which obviously is not possible under the intervalmeasure hypothesis. The latter hypothesis would, in general, expect the times of occurrence of both responses to depend upon $D$.

These considerations require one to measure response latencies as well as response probabilities.

\section{A Specific Model}

Since the experiments clearly show that the realtime criterion hypothesis holds and that the intervalmeasure hypothesis fails, a specific real-time criterion model will be developed in this section.

The time between $C$ and $B_{2}$ is $\gamma$ and

$$
\gamma=\left(\alpha_{2}+\mathrm{D}\right)-\left(\alpha_{1}+\mathrm{I}\right)
$$

Response choice is governed by $\gamma, \mathbf{R}(\mathrm{L})$ occurring when $\gamma>0$ and $R(S)$ when $\gamma<0$. The proportion of responses which are $R(L), P(L)$, therefore depends upon $\mathrm{D}, \mathrm{I}, \alpha_{1}$, and $\alpha_{2}$, and the psychophysical function, $P(L)=f(D)$, is determined by the two afferent latencies and $\mathrm{I}$. The variance of the psychophysical function, and thus the fineness of duration discrimination, is therefore, assuming independence,

$$
\mathrm{V}(\gamma)=\mathrm{V}(\mathrm{I})+\mathrm{V}\left(\alpha_{1}\right)+\mathrm{V}\left(\alpha_{2}\right)
$$

It would be unreasonable to assume that the two afferent latencies are identical, but there are grounds for the assumption that both of them have zero variance (Kristofferson, 1976). That assumption is made as part of this specific model, and for this model

$$
\mathrm{V}(\gamma)=\mathrm{V}(\mathrm{I}) \text {. }
$$

Variable error and the size of the duration threshold are determined solely by variability in the internal interval I. The theoretical distribution which generates the psychophysical function is simply the distribution of C, i.e., the distribution of times of occurrence of $C$ measured with respect to $P_{1}$.

The distribution of $C$ is shown in Figure 2. It is assumed to be an isosceles triangle with a base of $2 \mathrm{q}$ msec, since it has been shown for vision (Allan \& Kristofferson, 1974b; Allan, Kristofferson, \& Weins, 1971) and for audition (Kristofferson, Note 1) that such a form fits the psychophysical data very well. The cumulative form of this distribution is a fully bounded sigmoid function (Kristofferson \& Allan, 1973), which provides at least as good a fit as does a normal ogive (Allan et al., 1971). The convolution of two independent uniform distributions, each with a span of $\mathrm{q}$, generates the triangle, and $q$ is a unit of central temporal variability which has been called the "time quantum" (Kristofferson, 1967).

For stimulus $\mathrm{j}, \overline{\mathrm{P}}\left(\mathrm{L} / \mathrm{S}_{\mathrm{j}}\right)$ is the probability that $\mathrm{C}$ is less than $B_{2 j}$. The four duration stimuli yield $\mathbf{B}_{2}$

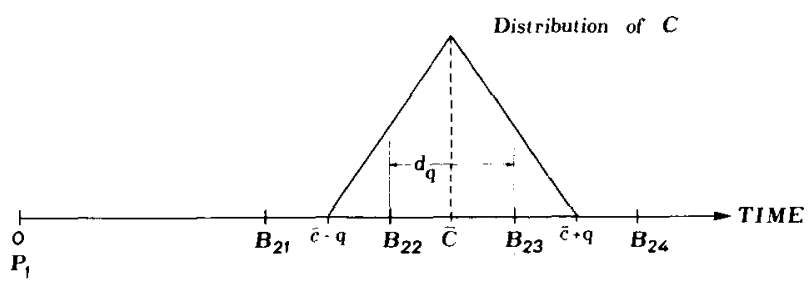

Figure 2. A specific model of the real-time criterion hypothesis. All discrimination errors are generated by variability in $\mathbf{C}$ which is assumed to have a distribution as shown: an isosceles triangle with base $=2 q$. $B_{2}$ is shown for each of four duration stimuli. $P(L)$ for $S_{i}$ is the proportion of area under the triangle to the left of $B_{2 i} \cdot \alpha_{2}$ has no variance, but $\alpha_{1}$ and $\alpha_{2}$ are not necessarily equal. $\bar{C}$ occurs at $\left(D_{c}+\alpha_{2}\right), D_{c}$ is the psychophysical criterion, and psychophysical range is defined as $(\bar{C} \pm q)$. 
values which cut the distribution at different points. The proportion of the area of the triangle to the left of $B_{2 J}$ is $\bar{P}\left(L / S_{j}\right)$.

For any two duration stimuli for which $B_{2}$ falls within the distribution, such as $S_{2}$ and $S_{3}$ in Figure 2, the obtained response probabilities are sufficient to calculate $d_{q}$, the distance between the two $B_{2} s$ in units of $q$ (Kristofferson \& Allan, 1973). If it is assumed that $\alpha_{2}$ is the same for the two stimuli, then $d_{q}$ in milliseconds will be equal to $\Delta D$, the difference in duration between $S_{2}$ and $S_{3}$, and

$$
\mathrm{q}=\frac{\Delta \mathrm{D}}{\mathrm{d}_{\mathrm{q}}}
$$

Thus, $q$ can be estimated from the response probabilities, as can $D_{c}$, the psychophysical criterion which is the duration of the stimulus for which $\mathrm{B}_{2}$ falls at the expected value of $C$.

The model, as developed up to this point, requires that $d_{q}$ be a zero-intercept linear function of $\Delta D$. Allan et al. (1971) found that to be the case. They also found, through an analysis of ROC curves, that the members of a stimulus set all appear to have the same psychophysical variance. Their results led them to propose the "onset-offset" theory of duration discrimination which contained the isosceles triangle as a formal representation. They concluded that (a) there is a perfect mapping of stimulus duration into psychological duration and (b) all psychophysical variance is associated with the afferent latencies.

The present model retains the formal representation but places all the variability in the criterion rather than in the stimulus. The stimuli appear to have the same variance merely because it arises from a source which is the same for all of them. The perfect mapping is due to the fact that everything, internal and external, occurs in real time and there is no transformation of stimulus time into psychological time.

Figure 2 shows that the outside members of the stimulus set, $S_{1}$ and $S_{4}$, are chosen to be outside the distribution. They should be discriminated perfectly and they are alway's included in a stimulus set for the purpose of monitoring the subject (Allan \& Kristofferson, 1974b).

Once $q$ has been estimated, the variance of $C$ can be calculated:

$$
\mathrm{V}(\mathrm{C})=\mathrm{V}(\mathrm{I})=\frac{\mathrm{q}^{2}}{6} .
$$

\section{Response Latencies in Speeded Duration Discrimination}

Extending the model to response latencies for the case in which the subject is asked to make his responses $R(S)$ and $R(L)$ as rapidly as possible causes several interesting features to emerge. Since $R(S)$ is a direct response triggered by $P_{2}$, it should, in the limit, yield a latency distribution which resembles the simple reaction time distribution. The distribution of $R(L)$ latencies should, in the limit, resemble a distribution of delayed reaction times or "time estimation" responses.

If we let $\lambda_{S}$ and $\lambda_{L}$ represent the response latencies [the time from $P_{1}$ to $R(S)$ and $R(L)$, respectively] for a stimulus of duration $D$, and define $K_{S}$ as an independent random variable to represent the time between $B_{2}$ and $R(S)$, and $K_{L}$ as the time between $C$ and $R(L)$, then

$$
\lambda_{\mathrm{S}}=\alpha_{2}+\mathrm{K}_{\mathrm{S}}+\mathrm{D}
$$

and

$$
\lambda_{\mathrm{L}}=\alpha_{1}+\mathrm{I}+\mathrm{K}_{\mathrm{L}} \text {. }
$$

The difference between the two latency variances for any $D$ is therefore

$$
\mathrm{V}\left(\lambda_{\mathrm{L}}\right)-\mathrm{V}\left(\lambda_{\mathrm{S}}\right)=\mathrm{V}(\mathrm{I})+\mathrm{V}\left(\mathrm{K}_{\mathrm{L}}\right)-\mathrm{V}\left(\mathrm{K}_{\mathrm{S}}\right) .
$$

For any $D$, the expected value of $\lambda_{S}$ (see Equation 6) is

$$
\mathrm{E}\left(\lambda_{\mathrm{S}}\right)=\alpha_{2}+\mathrm{E}\left(\mathrm{K}_{\mathrm{S}}\right)+\mathrm{D}
$$

and

$$
\mathrm{E}\left(\lambda_{\mathrm{S}}\right)-\mathrm{D}=\alpha_{2}+\mathrm{E}\left(\mathrm{K}_{\mathrm{S}}\right)
$$

is the mean latency of $R(S)$ measured from $P_{2}$ rather than $P_{1}$.

For all $\mathrm{D}$ greater than $\left(\mathrm{D}_{\mathrm{c}}+\mathrm{q}\right)$, since $\left[\alpha_{1}+\right.$ $\mathrm{E}(\mathrm{I})]=\left[\alpha_{2}+\mathrm{D}_{\mathrm{c}}\right]$, it follows from Equation 6 that the expected value of $\lambda_{\mathrm{L}}$ is the single value

$$
E^{\prime}\left(\lambda_{L}\right)=\alpha_{2}+D_{c}+E\left(K_{L}\right)
$$

in which the prime mark indicates the restricted range of $D$. Thus, within that range, i.e., $D>\left(D_{c}+q\right)$, $E\left(\lambda_{L}\right)$ is independent of $D$ and $E^{\prime}\left(\lambda_{L}\right)$ measured from $D_{c}$ rather than $P_{1}$ is

$$
E^{\prime}\left(\lambda_{L}\right)-D_{c}=\alpha_{2}+E\left(K_{L}\right) .
$$

The theoretical variables $K_{S}$ and $K_{L}$ each represent the total delay between one of the response triggers and its associated response. These response triggers, $C$ and $B_{2}$, are defined differently. However, they are in some sense juxtaposed by the requirement to discriminate and the two responses may be virtually identical. That $\mathbf{K}$ can sometimes be identical in variance for simple $\mathrm{RT}$ and time estimation responses 
has recently been demonstrated (Kristofferson, 1976). But that $K_{S}=K_{L}$ in the present context can only be a working assumption which leads to the expectation, from Equation 7, that $V\left(\lambda_{L}\right)-V\left(\lambda_{S}\right)=V(I)$ and further, from Equation 5, that

$$
\mathrm{V}^{\prime}\left(\lambda_{\mathrm{L}}\right)-\mathrm{V}(\lambda)_{\mathrm{S}}=\frac{\mathrm{q}^{2}}{6},
$$

in which $V^{\prime}\left(\lambda_{L}\right)$ is $V\left(\lambda_{L}\right)$ for $D \geqslant\left(D_{c}+q\right)$. Duration stimuli above the psychophysical range, viz, longer than $\left(D_{c}+q\right)$, should always elicit $R(L)$ and they should all have the same latency variance, a variance greater than $V\left(\lambda_{S}\right)$ by $q^{2} / 6$. Thus, from the variance difference of Equation 10, an estimate of $q$ can be obtained which should agree with that calculated from the response probabilities via Equation 4. Further, the distribution of $\lambda_{\mathrm{L}}$, in this range, should be the convolution of the distribution of $\lambda_{\mathrm{S}}$ with a triangular distribution of base $2 \mathrm{q}$.

For stimulus durations within the psychophysical range, $\left(D_{c}-q\right)<D<\left(D_{c}+q\right)$, $V\left(\lambda_{L}\right)$ is a function of $D$. Referring to Figure 2, when $B_{2}$ is within the distribution, those values of $C$ which are effective $R(L)$ triggers are those to the left of $B_{2}$. As $B_{2}$ moves leftward through the distribution, both the variance and the mean of the effective $\mathrm{C}$ decrease and as $\overline{(C}-q)$ is approached, the distribution of $\lambda_{L}$ approaches the distribution of $\lambda_{\mathrm{S}}$ in all respects.

Finally, it follows from Equations 8 and 9 that the mean latency of $R(S)$, measured from the onset of $P_{2}$, should be equal to the mean latency of $R(L)$ measured from the psychophysical criterion, and using stimuli for which $D>\left(D_{c}+q\right)$, when two conditions are met: (1) $\mathrm{E}\left(\mathrm{K}_{\mathrm{S}}\right)=\mathrm{E}\left(\mathrm{K}_{\mathrm{L}}\right)$ and (2) $\alpha_{2}$ has the same value when the two different response latencies are measured.

\section{Response Competition and Other Complications}

Both response triggers, $B_{2}$ and $C$, occur on every trial, but only one of the two responses occurs. Each trigger must be a negative signal for the opposite resporise as well as a positive signal for its own response. The first trigger must inhibit the response caused by the second trigger (both responses are never made on a trial, even when speeded), and it is probable that the inhibitory component of the second trigger will have some effects upon the response to the first trigger. The inhibitory effect of the second trigger upon the first must be zero when the interval between triggers is large, but it must increase as that interval becomes smaller, although not necessarily monotonically. If it is monotonic, then the amount of inhibitory effect should increase as the probability that the first trigger will be first decreases. For example, in Figure 2, when $S_{3}$ is presented, $B_{23}$ occurs before $C$ with small probability, but when it does so occur, $\mathrm{C}$ occurs very soon thereafter. Response competition should be maximum for $R(L)$ when $D=\left(D_{c}-q\right)$ and for $R(S)$ when $D=\left(D_{c}+q\right)$ rather than for stimuli near the criterion $D_{c}$ as might be expected on other grounds.

The decision rule which determines response choice states that the response associated with the first trigger is the response which occurs on every trial. That rule works well as far as discrimination probabilities are concerned and it will be retained. But it is possible that at least sometimes the response that occurs is the one associated with the second trigger and a response competition theory of duration discrimination might be formulated.

Response latencies are another matter. It seems quite possible that the latency of the response to the first trigger will be affected by the second trigger when the second occurs soon after the first. Extensive preliminary experiments showed this to be the case: there is much "response competition" in the latency data, especially when D is within the psychophysical range, when one of two possible overt responses must be made on each trial. Using a oneresponse procedure, in which in some sessions the response is $\mathrm{R}(\mathrm{L})$ and no overt response is made as $\mathrm{R}(\mathrm{S})$, while in other sessions that is reversed, greatly reduces the response competition effect. In those preliminary experiments, while latencies did depend upon whether one or two responses were required, discrimination performance did not. Such a result would suggest that discrimination errors are not due to response competition and careful systematic experiments are needed on this point.

Speeded duration discrimination was characterized above as a combination of simple RT and time estimation. As a simple RT experiment, it is one in which the RT signal occurs only on some trials, the subject must be generating $I$ at the moment the RT signal occurs, and the foreperiod varies. As a time estimation experiment, it is one in which the TE response is countermanded on some proportion of the trials. Given these considerations and what is known about RT and TE, it would indeed be remarkable if, in the limit, latency distributions could be obtained which resembled simple RT and TE.

The experiments which are reported next are intended to determine whether an interval measure strategy or a real-time criterion strategy is used in duration discrimination when a single stimulus, many-to-few method is employed. If the evidence is clear in favor of the real-time criterion strategy, then the predictions of the specific model developed above will be tested.

\section{METHOD}

An experimental session in Experiment 1 consisted of three blocks of 100 trials each with a brief pause between blocks. 
Within any session, the blocks were identical and the duration of a session was about $35 \mathrm{~min}$.

On each trial, one duration stimulus was presented and the subject attempted to classify it as "long" or "short." A single response procedure was used so that in some sessions the response was $R(L)$ while in other sessions it was $R(S)$. At the end of each trial, the subject was told whether the stimulus had been long or short. Each occurrence of a response was recorded by the computer along with its latency in milliseconds from the onset of the stimulus pulse which marked the beginning of the stimulus duration. The responses were buttonpresses, as described in detail elsewhere (Kristofferson, 1976).

Each trial began with a 100 -msec auditory warning signal followed 1,000 msec later by the duration stimulus. The duration stimulus consisted of two identical auditory pulses, $P_{1}$ and $P_{2}$, and the stimulus duration was the time $D$ between the onsets of $P_{1}$ and $P_{2}$, as shown in Figure 1 . The auditory pulses each had a duration of $10 \mathrm{msec}$. They were $1,000-\mathrm{Hz}$ sinusoids gated at zero crossing with a rise-decay time of $2.5 \mathrm{msec}$ and a loudness of $68 \mathrm{~dB}$ re 0.0002 dynes $/ \mathrm{cm}^{2}$ when on continuously. The stimulus patterns, responses, and computer program were the same as those used to study response-stimulus synchronization (Kristofferson, 1976). The only difference was that in the present experiment a visual feedback signal occurred at the end of the response period to tell the subject whether the duration had been "long" or "short."

In every session, there were four duration stimuli: $S_{1}$ and $S_{2}$ were designated short and $S_{3}$ and $S_{4}$ long. The one of the four to be presented on a trial was selected randomly, and they were equally likely to be selected. Thus each was presented about 75 times during a session in Experiment 1. All 300 trials were included in the final data analysis. $D_{1}$ and $D_{4}$ were set so that $S_{t}$ and $S_{4}$ were outside the psychophysical range and $S_{2}$ and $S_{3}$ were placed within the psychophysical range and separated by a $\Delta D$ of about one qunit.

Two experiments are reported below. Experiment 1 consisted of an intensive study of a single midpoint using a single subject, the author, who had had extensive prior experience. Two young adult males who had had no previous experience were the subjects for Experiment 2.

\section{EXPERIMENT 1}

Sets of four stimulus durations with a fixed mid- point of $1,150 \mathrm{msec}$ were used throughout this experiment. The inside members, $S_{2}$ and $S_{3}$, were never changed; they had durations of 1,100 and $1,200 \mathrm{msec}$, respectively. The durations of $S_{1}$ and $S_{4}$ were varied so that $B_{21}$ took various positions below $(\bar{C}-q)$ and $B_{24}$ covered a range above $(\bar{C}+q)$ (refer to Figure 2).

Some details of experimental design are given in Table 1. A total of 114 sessions were conducted, organized into 19 cycles of 6 sessions each. All changes in $D_{1}$ and $D_{4}$ were made between cycles, and the values of each are given in the table. For the first 5 cycles, the single response signaled the decision "short"; its meaning was reversed for the final 14 cycles.

The experimental subject had participated in similar duration discrimination experiments for several hundred sessions before beginning this one. These included many different midpoints with unspeeded responding followed by several different midpoints with speeded responding but with two responses. Immediately prior to the present experiment, he had done many sessions with midpoints close to the 1,150 used here but with two speeded responses. His discrimination performance was therefore specifically and highly trained for this experiment before it began.

Table 1 shows that performance was exceedingly reliable. When the monitoring stimuli $S_{1}$ and $S_{4}$ were presented, a small number of errors occurred during the first 5 cycles and a somewhat larger number for a few cycles following the reversal of response meaning at the beginning of Cycle 6. During the final 10 cycles $\left(18,000\right.$ trials), no errors were made on $S_{1}$ and $S_{4}$; the response always occurred when $S_{4}$ was presented and it never occurred when $S_{1}$ was presented.

The reversal of response meaning also affected

Table 1

Psychophysical Data for Experiment 1

\begin{tabular}{|c|c|c|c|c|c|c|c|c|c|}
\hline Cycle & $\mathbf{R}$ & $\mathrm{P}\left(\mathrm{L} / \mathrm{S}_{1}\right)$ & $P\left(L / S_{2}\right)$ & $\mathrm{P}\left(\mathrm{L} / \mathrm{S}_{3}\right)$ & $\mathrm{P}\left(\mathrm{L} / \mathrm{S}_{4}\right)$ & $\mathrm{D}_{1}$ & $\mathrm{D}_{4}$ & $\mathrm{D}_{\mathrm{c}}$ & $q$ \\
\hline 1 & $\mathbf{R}_{\mathbf{S}}$ & .004 & .156 & .935 & .996 & 1030 & 1270 & 1141 & 92.6 \\
\hline 2 & $\mathbf{R}_{\mathbf{S}}$ & .000 & .124 & .919 & .998 & 1030 & 1270 & 1146 & 90.9 \\
\hline 3 & $\mathrm{R}_{\mathrm{S}}$ & .000 & .171 & .948 & .998 & 1000 & 1300 & 1138 & 91.5 \\
\hline 4 & $\mathbf{R}_{\mathbf{S}}$ & .000 & .178 & .950 & 1.000 & 970 & 1330 & 1137 & 92.0 \\
\hline 5 & $\mathbf{R}_{\mathbf{S}}$ & .000 & .174 & .899 & .998 & 950 & 1350 & 1143 & 104.1 \\
\hline 6 & $\mathbf{R}_{\mathbf{L}}$ & .005 & .191 & .908 & .997 & 970 & 1330 & 1140 & 104.9 \\
\hline 7 & $\mathbf{R}_{\mathbf{L}}$ & .004 & .203 & .898 & .993 & 970 & 1330 & 1140 & 109.8 \\
\hline 8 & $\mathbf{R}_{\mathbf{L}}$ & .002 & .185 & .907 & 1.000 & 970 & 1330 & 1141 & 104.1 \\
\hline 9 & $\mathbf{R}_{\mathrm{L}}$ & .000 & .281 & .946 & .998 & 970 & 1330 & 1127 & 108.6 \\
\hline 10 & $\mathrm{R}_{\mathrm{L}}$ & .000 & .219 & .936 & 1.000 & 970 & 1330 & 1134 & 102.0 \\
\hline 11 & $\mathbf{R}_{\mathrm{L}}$ & .000 & .171 & .936 & 1.000 & 970 & 1330 & 1139 & 94.6 \\
\hline 12 & $\mathbf{R}_{L}$ & .000 & .213 & .933 & 1.000 & 970 & 1330 & 1135 & 101.9 \\
\hline 13 & $\mathrm{R}_{\mathrm{L}}$ & .000 & .166 & .933 & 1.000 & 970 & 1330 & 1140 & 94.5 \\
\hline 14 & $\mathbf{R}_{\mathbf{L}}$ & .000 & .144 & .906 & 1.000 & 970 & 1330 & 1145 & 97.2 \\
\hline 15 & $\mathrm{R}_{\mathrm{L}}$ & .000 & .127 & .907 & 1.000 & 970 & 1375 & 1147 & 93.9 \\
\hline 16 & $\mathrm{R}_{\mathrm{L}}$ & .000 & .282 & .942 & 1.000 & 970 & 1550 & 1127 & 110.1 \\
\hline 17 & $\mathbf{R}_{\mathbf{L}}$ & .000 & .201 & .924 & 1.000 & 970 & 1550 & 1138 & 102.5 \\
\hline 18 & $\mathbf{R}_{\mathrm{L}}^{\mathbf{L}}$ & .000 & .181 & .941 & 1.000 & 970 & 1550 & 1138 & 94.9 \\
\hline 19 & $\mathrm{R}_{\mathrm{L}}$ & .000 & .146 & .914 & 1.000 & 970 & 1550 & 1144 & 95.7 \\
\hline
\end{tabular}

Note-Each cycle is six consecutive sessions, hence each proportion is based upon 450 trials. $D_{1}$ and $D_{4}$ are the durations of $S_{1}$ and $S_{4} . D_{2}$ and $D_{3}$ were fixed at 1100 and 1200 , respectively. All times are in milliseconds. Subject A.K. 

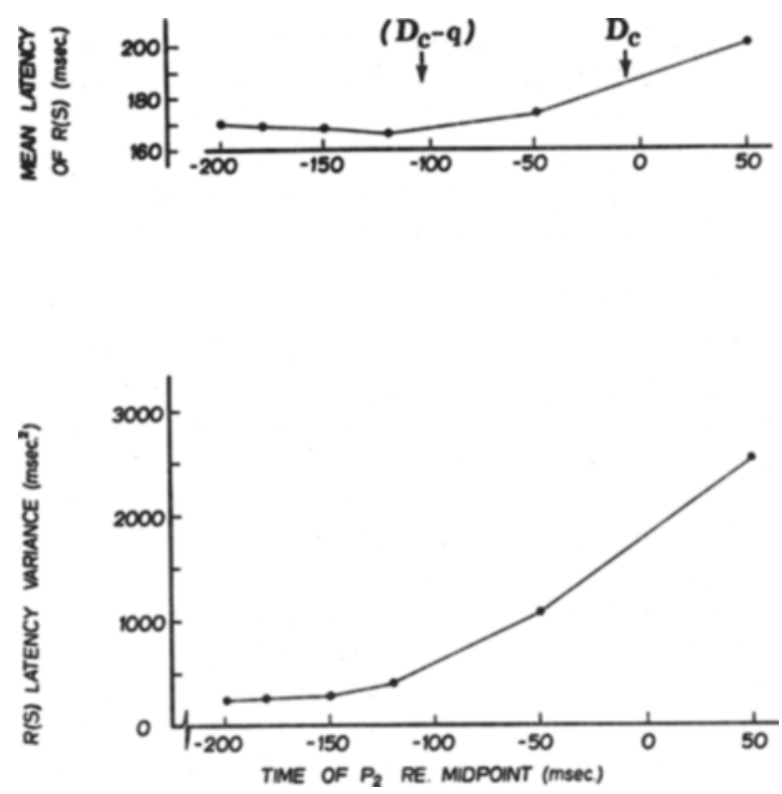

Figure 3. Mean and variance of $R(S)$ latencies, measured from $P_{2}$, as a function of the time at which $P_{2}$ is presented. Zero is $1,150 \mathrm{msec}$, the midpoint of the stimulus set. The lower half of the psychophysical range is the region between the arrows. Below that range, the mean latency is 168 , the $S D$ is 16.7 , and $R(S)$ is time-locked to $\mathbf{P}_{2}$.

latency variability. The variance of $R(L)$ latencies decreased slowly during Cycles 6-10. It remained constant thereafter until Cycle 16. Increasing $\mathrm{D}_{4}$ to 1,550 for Cycle 16 was accompanied by a small increase in latency variance, which diminished during cycles 16 and 17.

These minor perturbations following Cycle 5 and Cycle 15 also appear in Table 1 as small changes in the parameter q. For these reasons, Cycles 6-10 and 16-17 are omitted from the final analysis.

The remaining 12 cycles meet the stringent criteria of measurement: latency variances appear to be stationary and virtually no errors occur when one of the outside members of the stimulus set is presented. The mean value of the parameter $\mathrm{q}$ is $95.3 \mathrm{msec}$ during these 72 sessions. For the first 5 cycles, $q$ averages $94.2 \mathrm{msec}$. Thus, there is no indication here that discriminability depends upon which response is used.

\section{Response Latencies}

In Cycles 1-5, the time of occurrence of $R(S)$ was measured for each of six values of $D_{i}$. Figure 3 shows, in the upper graph, the mean response latency as measured from the onset of $P_{2}$ as a function of the time between $P_{2}$ and the midpoint of the stimulus set $(1,150 \mathrm{msec})$. The position of the psychophysical criterion and the point one $q$ below the criterion are indicated by arrows. The lower graph shows the corresponding variances.

When $P_{2}$ occurs more than one quantum before the criterion, $R(S)$ is strictly time-locked to $P_{2}$. The response occurs on average about $168 \mathrm{msec}$ after $P_{2}$ regardless of when $P_{2}$ is presented, and the variances average $279 \mathrm{msec}^{2}(\mathrm{SD}=17 \mathrm{msec})$.

Frequency distributions of $R(S)$ for $D$ equal to 950, 970, and 1,000 msec can be seen in Figure 4. They have a slight positive skew and a minimum near $135 \mathrm{msec}$. They are the same regardless of when $P_{2}$ is presented.

The means, variances, and distributions of $R(S)$ latencies are typical of those obtained in simple reaction time experiments under the most tightly controlled conditions (Kristofferson, 1976). All responses are shown in Figure 4; there are no outlying latencies. In the present experiment, $P_{2}$ occurs before $\left(D_{c}-q\right)$ on one-fourth of the trials. When it does so occur, $R(S)$ is certain to occur and $R(S)$ will occur within a 100 -msec window following $P_{2}$ regardless of when $P_{2}$ is presented within the range. Further, $R(S)$ never occurs on the one-fourth of trials on which $P_{2}$ is presented later than $\left(D_{c}+q\right)$.

It is concluded that short responses are, in the limit, simple reaction times triggered by $P_{2}$ and that under these rather complex experimental conditions these simple RT distributions are homogeneous in the sense that all of the responses are triggered by the action stimulus.

Frequency distributions of $R(S)$ latencies for $D$ equal to $1,030,1,100$, and $1,200 \mathrm{msec}$ are displayed in Figure 5. As $\mathbf{P}_{2}$ enters the psychophysical range, the skew becomes increasingly pronounced, but the minimum latency does not seem to change, staying about the same as in Figure 4. The minimum latency is time-locked to $P_{2}$ over the entire range.

While $R(S)$ is time-locked to $P_{2}, R(L)$ is not. The distributions of $R(L)$ when $D$ equals $1,330,1,375$, and 1,550, shown in Figure 6, demonstrate that $R(L)$ occurs at the same times following $P_{1}$ regardless of the time at which $P_{2}$ is presented, providing that $P_{2}$ is presented after $\left(D_{c}+q\right)$. In fact, $R(L)$ often

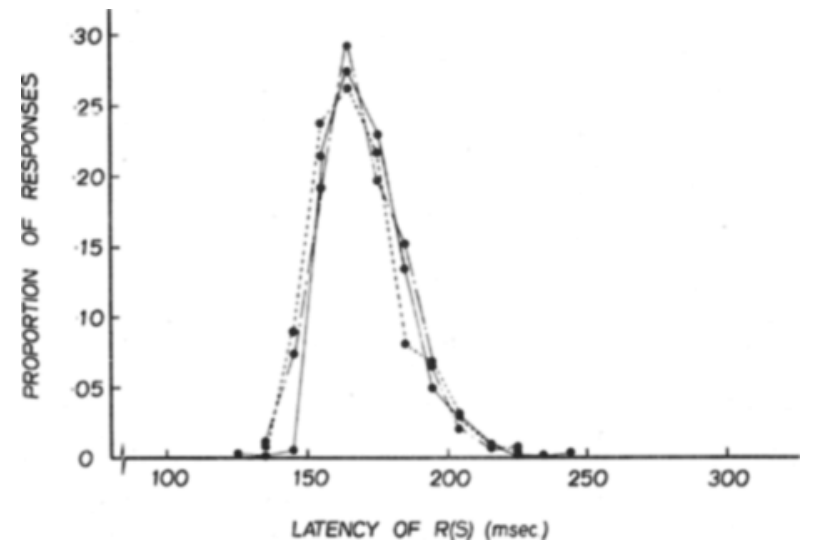

Figure 4. Frequency distributions of $\mathbf{R}(\mathbf{S})$ latencies for the three leftmost points of Figure 3 . Bin size $=10 \mathrm{msec}$. There are no outlying latencies. Latency measured re $P_{2} . R(S)$ is simple RT to $\mathbf{P}_{2}$. 


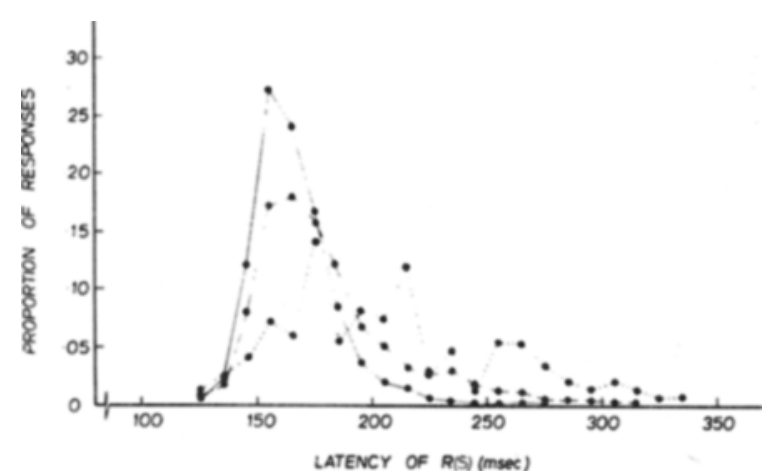

Figure 5. Frequency distributions for the three rightmost points of Figure 3. The minimum RT does not change, but the variance increases as $P_{2}$ moves upward within the psychophysical range. Latency re $\mathbf{P}_{2}$.

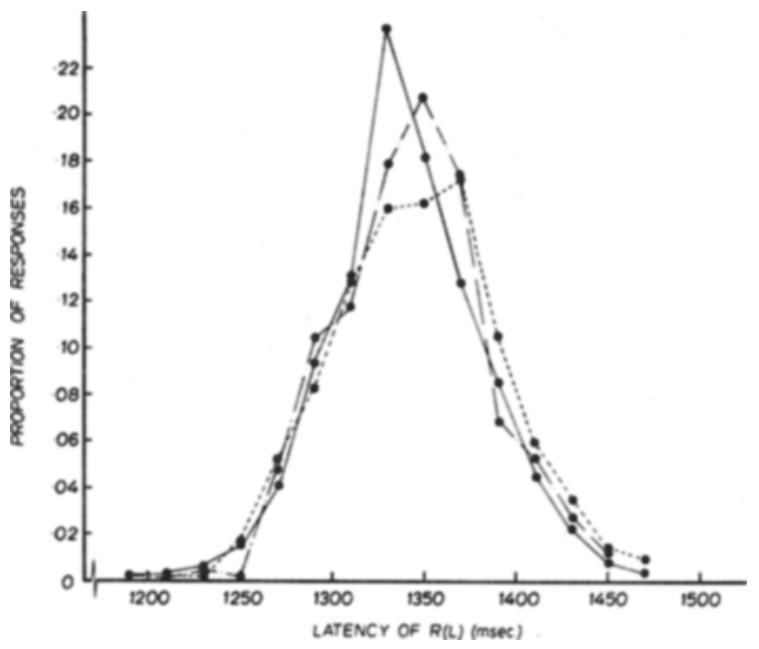

Figure 6. Frequency distributions of $R(L)$ latencies when $P_{2}$ is presented above the psychophysical range at 1,330 (solid line), 1,375 (dashed), and 1,550 msec (dotted). The means are 1,340, 1,343 , and 1,347 , and the variances are $1,704,1,703$, and 2,035 , in the same order. Bin size $=20 \mathrm{msec}$. No outlying latencies. The time of occurrence of $R(L)$ does not depend upon the time at which the stimulus duration ends. Latency re $P_{1}$.

occurs before $P_{2}$; in the extreme case of $D=1,550$, it always does. Since in addition, $R(L)$ never occurs when $S_{1}$ is presented, it is concluded that $R(L)$ is never triggered by $P_{2}$.

According to the specific model developed in the introduction, when $\mathbf{P}_{\mathbf{2}}$ is presented within the psychophysical range, the distribution of $R(L)$ should be truncated: the longer latencies should disappear but the shorter latencies should be unaffected. Figure 7 shows this truncation effect. As $\mathbf{P}_{2}$ moves downward toward $\left(D_{c}-q\right)$, the mean and the variance of $R(L)$ do diminish. For this subject, the alignment of the left edges of the distributions is not perfect; the truncated distributions shift to the left by a small amount which increases with the depth of the truncation.

\section{Response Latencies and Response Probabilities}

Within the psychophysical range $\left(D_{c} \pm q\right)$, response latencies yield an unclear picture, probably due to the temporal proximity of $C$ and $B_{2}$. But outside that range, the latencies are well behaved. According to Equation 10, the variance of $R(L)$ above the range should be greater than the variance of $R(S)$ below the range by $q^{2} / 6$. The mean value of $q$, calculated from the response probabilities for $S_{2}$ and $S_{3}$, is $95.3 \mathrm{msec}$. $V(C)$ is therefore, $1,512 \mathrm{msec}^{2}$. The difference between the two latency variances, calculated from the latencies of $S_{1}$ and $S_{4}$ for the same 12 cycles as was $\mathrm{q}$, is $1,799-279=1,520 \mathrm{msec}^{2}$. Put differently, the size of the quantum can be calculated from either the discrimination probabilities or the latency variances, and the result is the same, for this subject.

The mean latency of $R(S)$, measured with respect to $P_{2}$ in Cycles $1-5$, is $168 \mathrm{msec}$ for those $D$ below the psychophysical range. During Cycles 11-15 and 18-19, the mean value of the psychophysical criterion is $1,141 \mathrm{msec}$ and the mean latency of $R(L)$ is 1,342 msec when $D$ is above the psychophysical range. The difference between these is $201 \mathrm{msec}$, substantially more than 168 . Referring back to Equations 8 and 9 and the later discussion of them, it is necessary to conclude either that $E\left(K_{S}\right)$ is not equal to $E\left(K_{L}\right)$ or that $\alpha_{2}$ is different in the later cycles from what it is in the earlier cycles, or both.

\section{EXPERIMENT 2}

Two naive subjects, J.G. and P.W., were studied in this experiment, with the midpoint of the stimulus set fixed at $850 \mathrm{msec}$ for both of them. The values of $S_{1}$ and $S_{4}$ were 730 and $970 \mathrm{msec}$ for both, except as noted below, while $S_{2}$ and $S_{3}$ were always 800 and 900 for J.G. and 820 and 880 for P.W. Each session consisted of four blocks of 100 trials each, and four consecutive sessions comprise a cycle. J.G. did 15 cycles and P.W. did 16 .

J.G. used $R(L)$ as his single response for the first 7 cycles. He then switched to $R(S)$ for the remainder of the experiment. The reverse order was used for P.W.-R(S) for 9 cycles followed by $R(L)$ for 7 cycles.

For J.G., $S_{4}$ alternated between 970 and $1,030 \mathrm{msec}$ from session to session during Cycles 6 and 7 while $S_{1}$ alternated between 720 and $740 \mathrm{msec}$ during Cycles 13 and 14. For P.W., $S_{1}$ alternated between 720 and 740 during Cycles 7 and 8 while $S_{4}$ alternated between 950 and 990 during Cycles 15 and 16 .

During the first 4 cycles for J.G. and the first 3 cycles for $P$.W., the subjects were allowed to respond when they wished, no mention being made of speeded responding or of the fact that latencies were being measured. They were thereafter instructed to respond as rapidly as possible and given information about their latencies at the end of each session. 


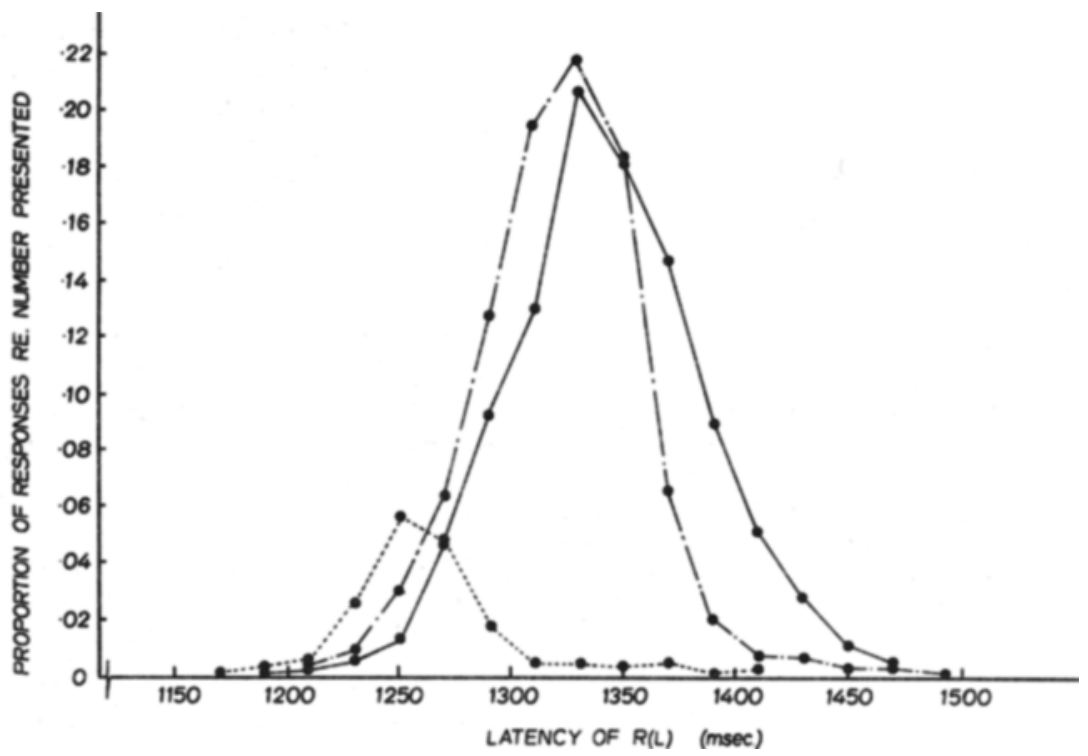

Figure 7. Truncation of the $R(L)$ distribution when $P_{2}$ falls within the psychophysical range. Proportions based upon number of stimulus presentations; thus when $P_{2}$ occurs at 1,100 msec (dotted line), $R(L)$ is less likely to occur, but when it does occur it does so more rapidly (mean $=1,261$ ) and with less variability (Variance $=932$ ). The dashed line is the distribution for $D_{3}=1,200$; its mean and variance are 1,323 and 1,234 . The solid line is the composite of the three distributions of Figure 6 .

\section{Response Probabilities}

A summary of the parameters obtained from the response probabilities is given in Figure 8 for $S_{2}$ and $S_{3}$ in the upper graph and for $S_{1}$ and $S_{4}$ in the lower. For both subjects, obtained values of $q$ were substantially higher when the response was $R(L)$ than when it was $R(S)$.

Instructions to respond rapidly affected latencies greatly, of course. For J.G., R(L) for $\mathrm{S}_{4}$ occurred at a mean time of $1,260 \mathrm{msec}$ following $P_{1}$ in Cycle 4; in Cycle 5, that latency decreased to $1,050 \mathrm{msec}$. For P.W., when $S_{1}$ was presented in Cycle 3, R(S) occurred at a mean time of $1,060 \mathrm{msec}$ while in Cycle 4 it occurred at $925 \mathrm{msec}$. Despite these gross changes in latency, discrimination accuracy was not noticeably affected by speeding, although, of course, the design of this experiment does not include the features required to make this a firm conclusion.

Both subjects made errors on $S_{1}$ and $S_{4}$ in every cycle of the experiment. The error rate, $\varepsilon$, has been interpreted as the probability that the stimulus is not processed when it is presented and that the response is due to some other cause in such cases (Allan \& Kristofferson, 1974b). The error rates are not large, but the ideal of zero is not achieved here as it was in Experiment 1. Even a small error rate of this kind suggests that the latency distributions will not be homogeneous. The error levels, too, were unaffected by speeding the responses.

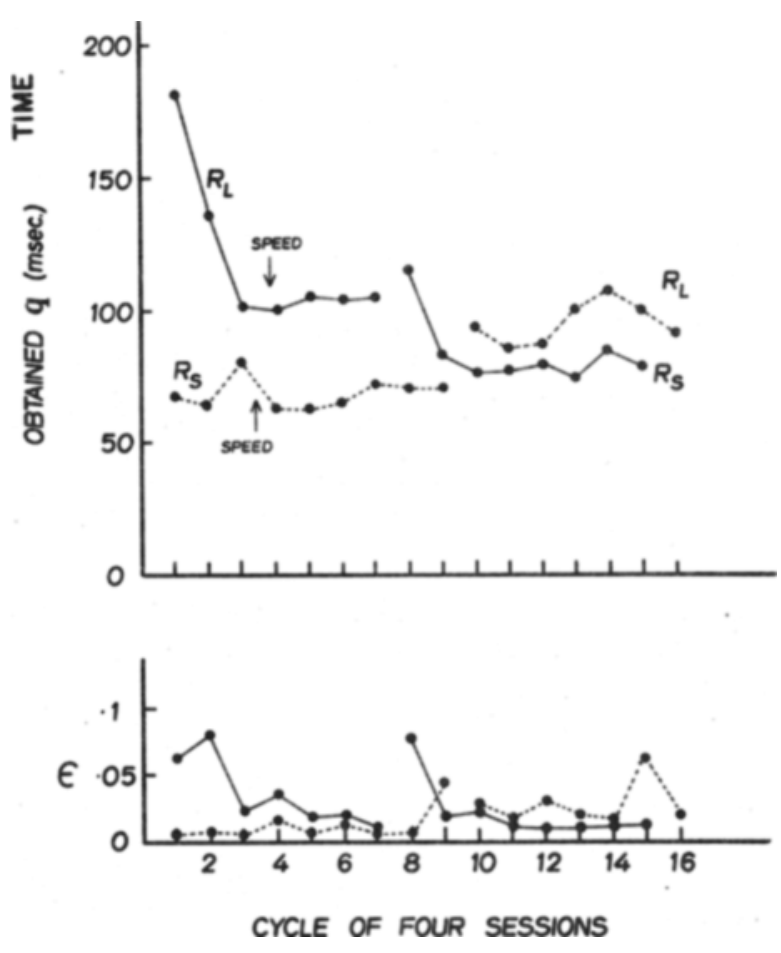

Figure 8. Duration discrimination results from Experiment 2. The upper graph shows values of $q$ calculated from $P(L)$ for $S_{2}$ and $S_{3}$ trials. The lower graph is of the error rate recorded on $S_{1}$ and $S_{4}$ trials: $\varepsilon=(1-K)=1-P\left(R_{L} / S_{4}\right)+P\left(R_{L} / S_{1}\right)$. Subject J.G. yielded the solid lines, P.W. the dotted lines. 


\section{Response Latencies}

The latencies of $R(S)$ on $S_{1}$ trials did not reach and maintain a stable level for either subject. This is evident in Figure 9. Both the mean and the variance reach their lowest levels in the final cycle for each subject, and it is likely that they would have diminished even more.

During the final cycle, the means were 191 and $164 \mathrm{msec}$ following $P_{2}$ for J.G. and P.W., respectively; the variances are 474 and $384 \mathrm{msec}^{2}$. These are close to the values obtained in Experiment 1 and all of them are well within the typical simple $\mathrm{RT}$ range.

The time of occurrence of $P_{2}$ was alternated between 720 and $740 \mathrm{msec}$ from session to session during the two cycles just before the final cycle for both subjects. The results are shown by the frequency distributions in Figures 10 and 11. It is apparent that delaying $P_{2}$ by $20 \mathrm{msec}$ shifts the response distribution to the right by about the same amount, $24 \mathrm{msec}$ for J.G. and 28 for P.W.

These distributions resemble simple RT distributions, as was the case also in Experiment 1. However, in Experiment 2, there are a few scattered latencies that are far from the mean, as might be expected from the nonzero values of $\varepsilon$.
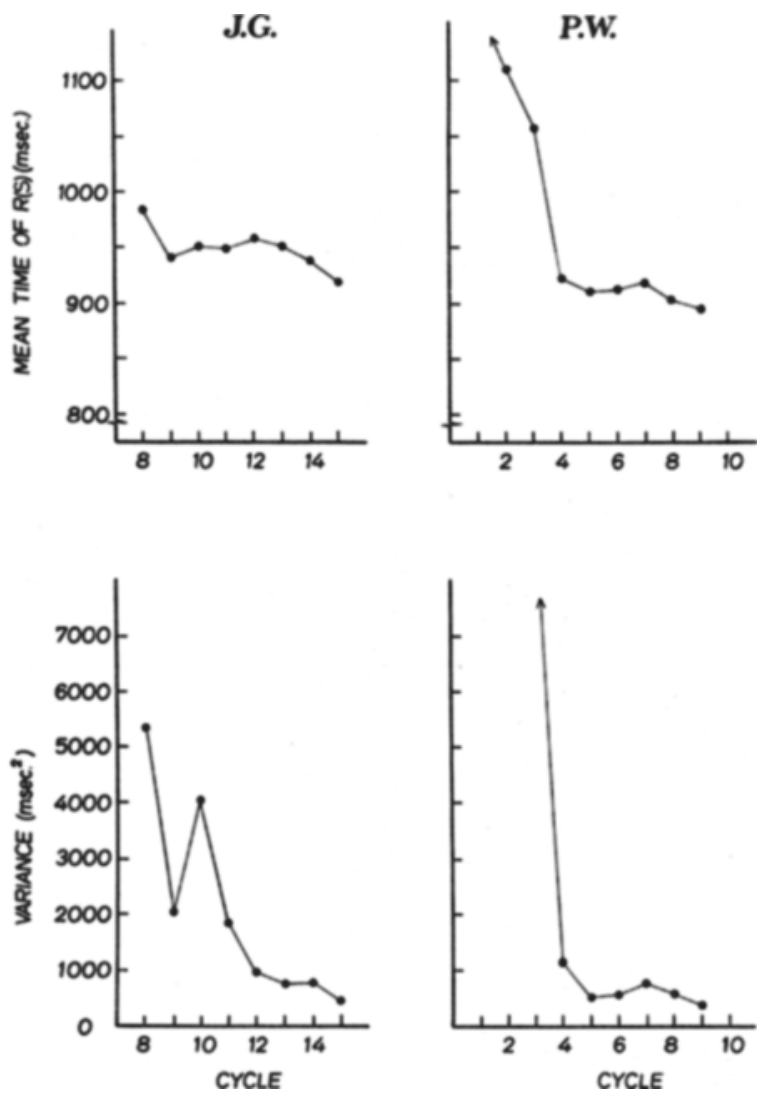

Figure 9. Latency means relative to $P_{1}$, and variances, for $R(S)$ on $S_{1}$ trials for the two subjects of Experiment 2.

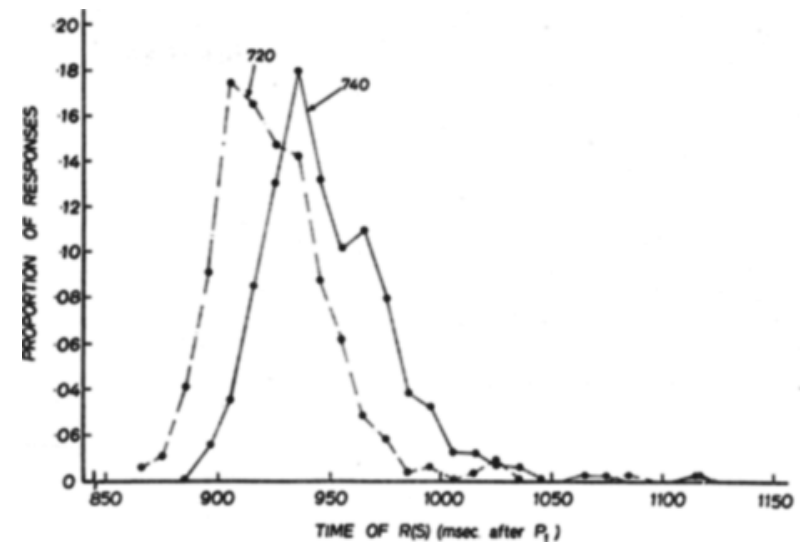

Figure 10. Frequency distributions of $R(S)$ on $S_{1}$ trials for $D_{1}=720$ and 740 msec. Subject J.G.

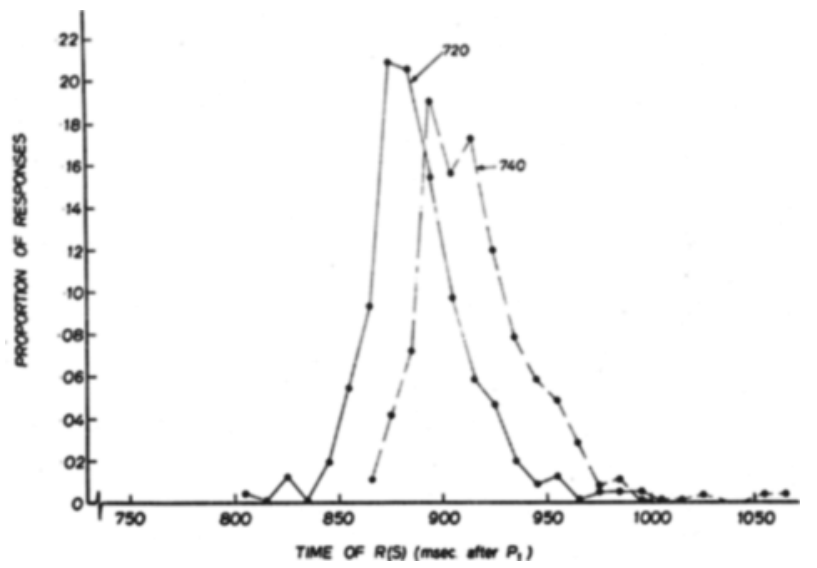

Figure 11. Frequency distributions of $R(S)$ on $S_{1}$ trials for $D_{1}=720$ and 740 msec. Subject $P$.W.

Like the $R(S)$ latencies, the $R(L)$ latencies did not reach and maintain a stable level in the course of this experiment. During the final two $R(L)$ cycles, the duration of $\mathrm{S}_{4}$ alternated from session to session between two values for each subject. The result is given in the form of frequency distributions in Figure 12, and the findings of Experiment 1 are confirmed. Many $R(L)$ occur before $P_{2}$, and the mean latency of $R(L)$ is independent of the time at which $P_{2}$ is presented.

The predicted truncation of the $R(L)$ distribution by $S_{2}$ and $S_{3}$ is even more clearly evident here (Figures 13 and 14) than it was in Experiment 1. For both subjects, the left edges of the distributions are unaffected by the truncation. The truncation is clean for J.G. but it is not for P.W., who has a long, positive tail for both $S_{2}$ and $S_{3}$.

\section{Response Latencies and Response Probabilities}

Because of the nonzero error rates and nonstationarity of the latencies in this experiment, it is pointless to consider the relations between probabilities and latencies in fine detail. 

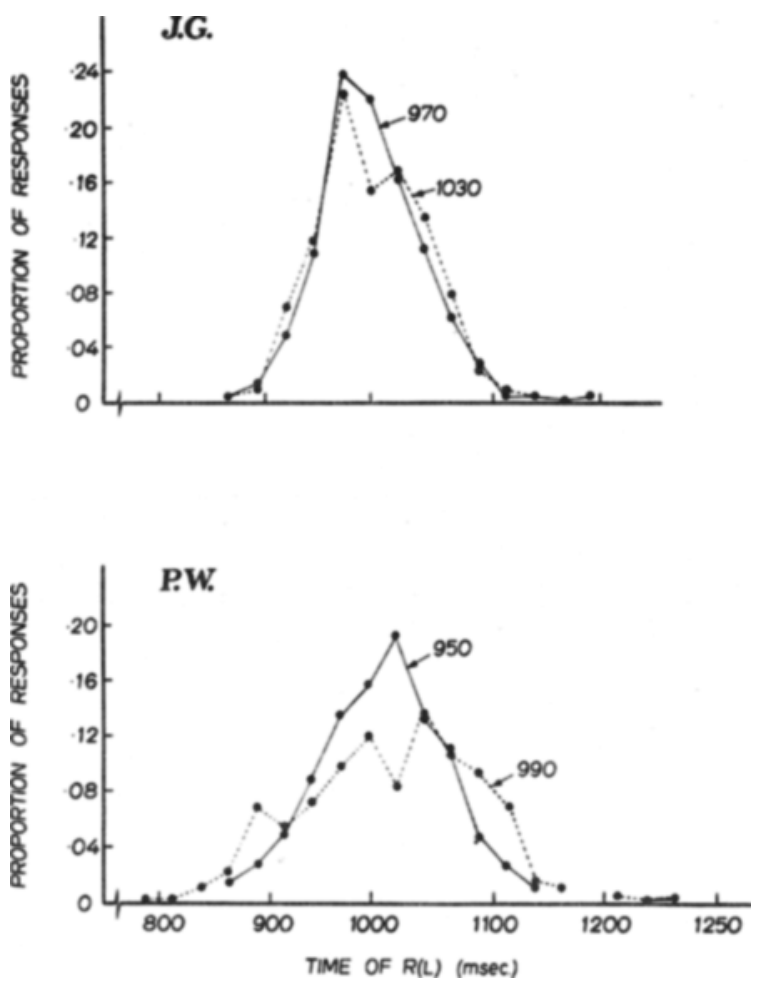

Figure 12. Frequency distributions of $R(L)$ for two values of $\mathrm{D}_{4}$ (as indicated) for each of the two subjects of Experiment 2. For the top graph, mean $=989$ and 991 and variance $=2,262$ and 2,334 for 970 and 1,030, respectively. For the lower graph, mean $=1,001$ and 1,006 and variance $=3,113$ and 5,528 for 950 and 990 , respectively.

The latency variance of $R(L)$ is much greater than that of $R(S)$, and the latter is similar to that of simple RT. The variance difference is somewhat larger than in Experiment 1, but had greater experimental control been achieved, that difference would probably be reduced.

The mean latencies of $R(S)$ are 191 and $164 \mathrm{msec}$ re $P_{2}$ for J.G. and $P$.W., respectively. The means for $R(L)$ are 161 and $178 \mathrm{msec}$, in the same order, re $D_{c}$.

\section{DISCUSSION}

Analyzing a duration stimulus, and discriminating between duration stimuli using the responses $R(L)$ and $R(S)$, is accomplished by a real-time criterion mechanism. The alternative, that a measure is taken of the stimulus duration over its temporal extent and the decision is based on such a measure, is rejected. Instead, the beginning of the duration stimulus triggers an internal time interval which runs along with the stimulus duration. If the internal interval ends first, the response $R(L)$ is selected and elicited. But if the sensory signal that is the end of the duration stimulus is received before the internal interval has ended, then $R(S)$ is the consequence.
The response $R(S)$ is caused by $P_{2}$, the end of the duration stimulus. Under ideal conditions, as in Experiment 1, it always occurs when it should $\left(\mathrm{S}_{1}\right.$ trials) and never when it should not ( $S_{4}$ trials). When it does occur, it does so within a narrow time window following $\mathrm{P}_{2}$ by an amount that is fixed and independent of the times at which $P_{2}$ occurs. $R(S)$ is time-locked to the end of the duration stimulus. The other response, $R(L)$, is quite the opposite. When it is certain to occur, the time at which it occurs is unrelated to $P_{2}$ and, in fact, the overt $R(L)$ may occur even before the end of the duration stimulus.

Both experiments confirm the foregoing in detail in every case, and it is concluded that short responses are caused by the end of the duration stimulus and long responses are caused by the beginning of the duration stimulus. The proof of this conclusion is strong but it is not yet complete. Additional experiments are required to establish that $R(L)$ is timelocked to $P_{1}$ and that $R(S)$ is not.

Speeded responding was required in these experiments, and it is possible that that forces a subject into a real-time criterion mode of processing. Some evidence is given that discrimination performance is unaffected by speeding the responses, and that supports the view that the real-time criterion is also used when responding is done at leisure, but it does not prove it.

Certain deductions from the general conclusion are also confirmed and add weight to it. One is that

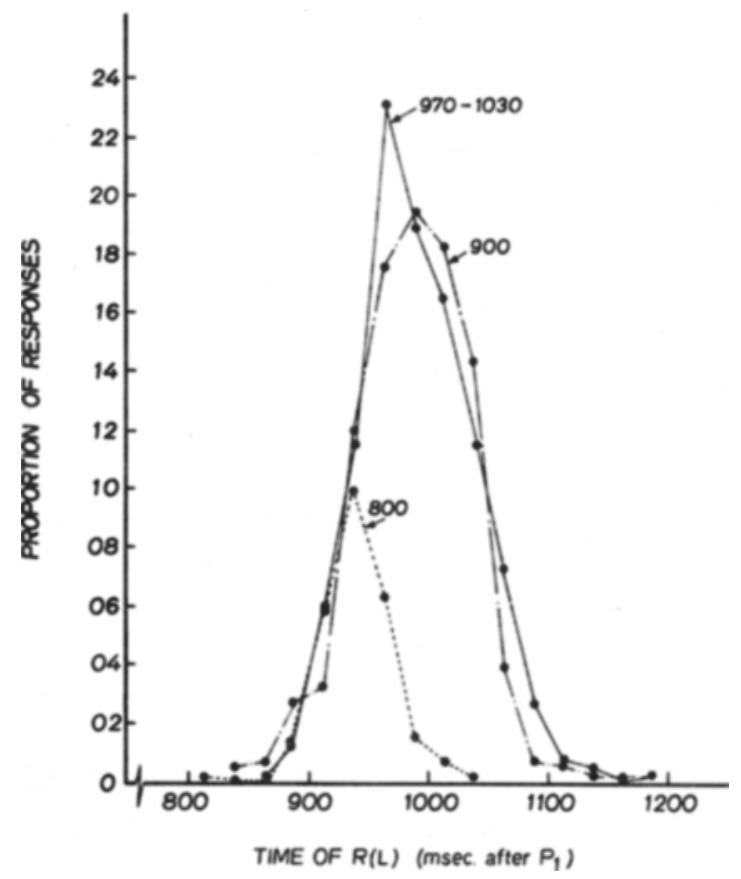

Figure 13. Truncation of the $R(L)$ distribution by $P_{2}$ occurring within the psychophysical range at $800\left(S_{2}\right)$ and $900 \mathrm{msec}\left(S_{3}\right)$. The solid line is the composite of the two distributions obtained on $S_{4}$ trials. Subject J.G. 


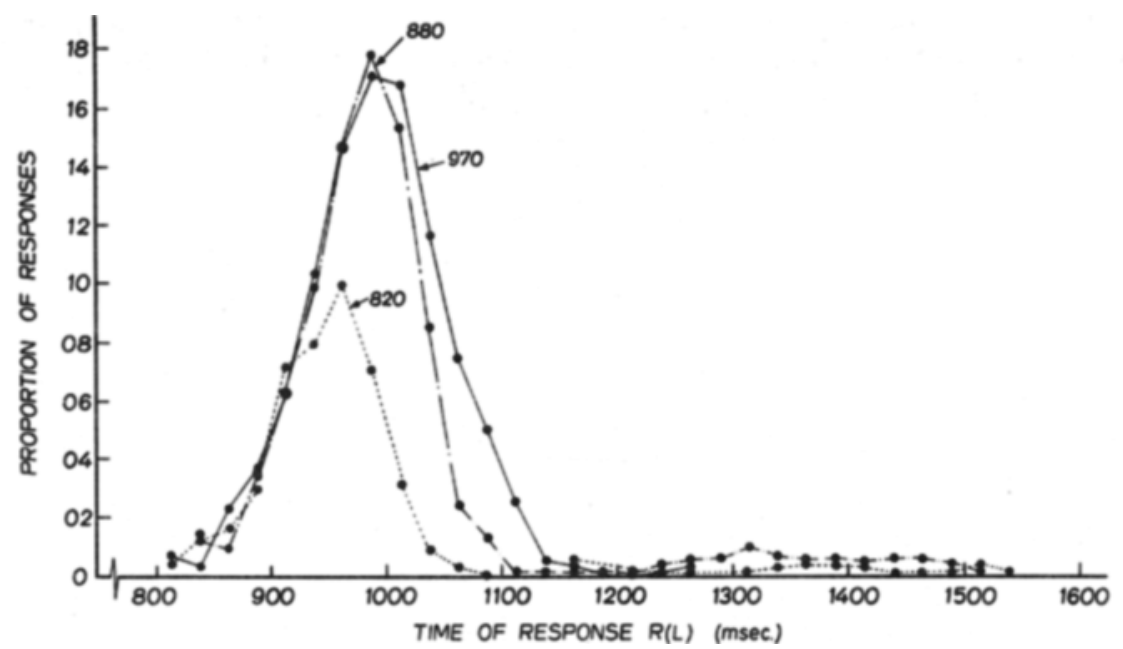

Figure 14. Truncation of the $R(L)$ distribution by $P_{2}$ occurring within the psychophysical range at $820\left(S_{2}\right)$ and 880 msec $\left(S_{3}\right)$. The solid line is the distribution obtained on $S_{4}$ trials when $D_{4}$ was 970 . Subject P.W.

$R(S)$ should resemble simple reactions to $P_{2}$. That is found to be true in every case: the latencies of $R(S)$ form distributions which are typical in all respects of simple RT distributions (for a discussion of what is "typical," see Kristofferson, 1976). Another deduction is that, since $R(L)$ is a response initiated by $P_{1}$, but only after an interpolated delay, the distributions of $R(L)$ latencies should resemble distributions of "time estimation" responses. This too is borne out: in Experiment 1, the ratio of SD to mean for $R(L)$ is 0.03 . That ratio and the shape of the distributions agree well with the time estimation data reported earlier (Kristofferson, 1976, especially in Figure 13).

Thus there are two causal chains to consider on a duration discrimination trial: $P_{1} \rightarrow R(L)$ and $P_{2} \rightarrow R(S)$. Since response choice depends upon whether an event $C$ in the $P_{1}$ chain occurs before or after an event $B_{2}$ in the $P_{2}$ chain, and since the psychophysical variance is much greater, usually, than the total latency variance of the $P_{2}$ chain, it might have been deduced that most of the psychophysical variance in duration discrimination is in the $P_{1}$ chain preceding $\mathrm{C}$. That is, errors in duration discrimination are due mainly to variability in the internal criterion interval.

In fact, the specific model tested above goes even further and proposes that all of the variability in duration discrimination is due to variability in the time at which $C$ occurs. The time between $P_{2}$ and its sensory effect, $B_{2}$, is assumed to be deterministic. In Experiment 1, where the measurement criteria are met, this model is confirmed; the same variance of $C$, and hence the same value of the parameter $q$, are recovered from both the latency variance and the response probabilities. In Experiment 2, in which stable latency levels were not shown to be achieved and the error rate on $\mathrm{S}_{1}$ and $\mathrm{S}_{4}$ trials was nonzero, only a qualitative agreement with the model can be seen.

The specific model is also supported by the relatively sharp truncation of the $R(L)$ distribution which occurs when $P_{2}$ is presented within the psychophysical range. This cutoff, which is already blurred by the variance in $K$, would be blurred further to the extent that $\alpha_{2}$, the afferent latency of $P_{2}$, is variable. It would be possible to determine theoretical truncated distributions by calculating the convolution of the truncated triangular section with the $R(S)$ distribution, but this would gain little because of the obvious presence of some degree of latency interaction between $C$ and $B_{2}$ when they occur in temporal proximity within the psychophysical range. It would be useful if one wished to study the response competition effects, but for present purposes this too must be left as a qualitative finding.

One other result from Experiment 2 requires some comment. Discrimination performance, as indexed by the parameter $q$ calculated from the response probabilities, is substantially poorer when the single response is $R(L)$ than when it is $R(S)$ (see Figure 8 ). Table 1 shows that this was true also in Experiment 1 during the first 30 sessions using $R(L)$. But in Experiment 1, in which sessions were continued until the latencies had definitely stabilized, the difference in $q$ between $R(S)$ and $R(L)$ conditions did not exist after stability was attained.

There is a difference between the $R(S)$ and $R(L)$ conditions which is inherent in the procedure and which may account for the greater difficulty in attaining stability when $R(L)$ is the response. When a response is made, the sound produced by the response can be heard. This response sound, perceived in relation to $P_{2}$, functions as a highly efficient 
feedback signal under certain conditions (Kristofferson, 1976). When the response being made is $R(S)$, the response sound follows $P_{2}$ on every trial by a substantial amount. But when $R(L)$ is used, the response sound may often occur close in time to $P_{2}$ when $S_{4}$ is presented, depending upon the value of $D_{4}$, and attempts to synchronize the response sound with $\mathrm{P}_{2}$ may conflict with the psychophysical discrimination, thereby inflating the variance of $C$. If this is so, there should be a critical value of $D_{4}$ that is compatible with $D_{c}$ and a stimulus set containing such an $\mathrm{S}_{4}$ should require much less practice to achieve stability.

There is a prediction of the specific model which will provide another strong test of it. Changes in base duration, i.e., in the midpoint of the stimulus set, should cause changes in discriminability, $q$, which are accompanied by quantitatively identical changes in the latency variances of $R(L)$ and by negligible changes in the latency variance of $R(S)$. Such changes should occur if the changes in midpoint are large, but there may be little change if they are small (Allan \& Kristofferson, 1974b).

Kristofferson (1976) has demonstrated an S-R causal chain which contains an adjustable and deterministic temporal segment. He suggested that that segment might be within the afferent latency. If so, and if it is within $\alpha_{1}$, as defined here, then the specific real-time criterion model would predict that in the limit there should be a range, at the lower end of the base duration scale, within which duration discrimination is independent of base duration and also virtually perfect. The experiment needs to be done to determine whether such a limit exists, there being no evidence of "virtually perfect" discrimination in past experiments.

Finally, what of the interval-measure hypothesis? ${ }^{3}$ Its rejection obviously does not mean that a measure of a stimulus duration cannot be taken, coded, and stored in memory. The real-time criterion mechanism itself suggests that this is possible and also that the memory representation can be decoded into a real, if internal, time interval. What is rejected is the hypothesis that an order decision about a stimulus duration is based upon a comparison between a coding of the presented stimulus and a representation held in memory. It may be that under some circumstances such does occur, but that it does now must be demonstrated. Such judgments about duration, based upon comparison of interval measures, may follow different laws than those based upon real-time processing, but we cannot know until the former have been shown to be isolated.

However, it is not even necessary to assume that these internal time intervals are stored in "coded" form. It is possible that they are simply the real duration of automatic, highly stable, memorial chains of the kind proposed by Schneider and Shiffrin (in press).

\section{REFERENCE NOTE}

1. Kristofferson, A. B. Psychological timing mechanisms. A lecture presented to the Lake Ontario Vision Establishment, Niagara Falls, Ontario, 9 March 1973. Mimeographed.

\section{REFERENCES}

Allan, L. G. The relationship between judgments of successiveness and judgments of order. Perception \& Psychophysics, 1975, 18, 29-36.

Allan, L. G., \& Kristofferson, A. B. Psychophysical theories of duration discrimination. Perception \& Psychophysics, 1974, 16, 26-34. (a)

Allan, L. G., \& Kristofferson, A. B. Judgments about the duration of brief stimuli. Perception \& Psychophysics, 1974, 15, 434-440. (b)

Allan, L. G., Kristofferson, A. B., \& Wiens, E. W. Duration discrimination of brief light flashes. Perception \& Psychophysics, 1971, 9, 327-334.

Creelman, C. D. Human discrimination of auditory duration. Journal of the Acoustical Society of America, 1962, 34, 582-593.

EIsLer, H. Subjective duration and psychophysics. Psychological Review, 1975, 82, 429-450.

KRISTOFFERSON, A. B. Low-variance stimulus-response latencies: Deterministic internal delays? Perception \& Psychophysics, 1976, 20, 89-100.

Kristofferson, A. B. Attention and psychophysical time. Acta Psychologica, 1967, 27, 93-100.

Kristofferson, A. B., \& Allan, L. G. Successiveness and duration discrimination. In S. Kornblum (Ed.), Attention and performance IV. New York: Academic Press, 1973. Pp. 737-749.

MAsSaRo, D. W., \& IDSON, W. L. Temporal course of perceived auditory duration. Perception \& Psychophysics, 1976, 20, 331-352.

SchneIDer, W., \& Shiffrin, R. M. Controlled and automatic human information processing: I. Detection, search and attention. Psychological Review, in press.

STUBBs, D. A. Scaling of stimulus duration by pigeons. Journal of the Experimental Analysis of Behavior, 1976, 26, 15-25.

Thomas, E. A. C., \& Weaver, E. B. Cognitive processing and time perception. Perception \& Psychophysics, 1975, 17, 363-367.

\section{NOTES}

1. Our review (Allan \& Kristofferson, 1974a) pointed out that all three of the quantitative theories that existed at that time assumed that the input process for a duration stimulus could not be complete until the stimulus had ended, and therefore that correct decisions and responses could not occur until after stimulus termination. One theory (Creelman, 1962) states that hypothetical random pulses are counted while the stimulus is present and that decisions are based upon the total count. The second theory is similar, except that time quanta of fixed duration are counted (see Allan \& Kristofferson, 1974a, for references). The third theory is the onset-offset model (Allan \& Kristofferson, 1974b), which is discussed later in the present paper.

Thomas and Weaver (1975) have proposed a theory of perceived duration which they have applied to mapping two brief stimulus durations into three response categories. Their theory states that perceived duration, upon which discrimination is 
assumed to depend, receives a contribution to its magnitude from a timer which measures the temporal extent of the stimulus. They restrict their theory to stimulus durations less than $100 \mathrm{msec}$, but that restriction is imposed not because the operation of the timer is thought to be different for longer durations.

Massaro and Idson (1976) have also proposed a theory of perceived duration which they apply to the discrimination between two brief durations using two responses. The theory is similar to the Thomas and Weaver theory, both being multiple-component theories which make perceived duration a function of variables in addition to stimulus duration. Massaro and Idson point out some differences between their theory and that of Thomas and Weaver. Massaro and Idson state that discrimination increases as the difference in perceived duration of the two stimulus durations increases. Perceived duration begins to grow at stimulus onset and the rate of growth depends upon the duration of the stimulus. It is difficult to understand how that can be so, since the information necessary to set the rate parameter cannot be available to the system until after stimulus offset. For that reason and others, the present author cannot determine whether theirs is an interval-measure hypothesis or how their theory would apply to longer stimulus durations.

Hannes Eisler (1975) presents a theory of subjective duration which encompasses psychophysical scaling of duration and duration discrimination within the same framework. He states that duration discrimination depends upon a comparison of two subjective durations. Each subjective duration is the total accumulated content of a hypothetical sensory register which integrates over the time of the duration stimulus. This is in an interval-measure hypothesis, and Eisler applies it to long and short durations.

2. When the stimulus set is symmetrical around its midpoint, errors are minimized when the criterion is placed at the midpoint. That is what subjects do, with great accuracy. If the set is made asymmetrical by, say, lengthening $D_{4}$, then the midpoint between $S_{2}$ and $S_{3}$ should still control the placement of the criterion, providing $S_{1}$ and $S_{4}$ are both far enough from that midpoint to give error-free performance. Subjects can also do that with considerable success, but sometimes the asymmetry does have an effect upon the placement of the criterion. But even with a symmetrical set, the criterion can easily be shifted away from the midpoint by, for example, calling $S_{4}$ "long" and the other stimuli "short." The criterion is then placed between $S_{3}$ and $S_{4}$. It is more accurate to say that "base duration" is the psychophysical criterion as measured for any particular combination of experimental conditions.

Experiments by Stubbs (1976) suggest the interpretation that when only two stimulus durations comprise the set, one much longer than the other, a criterion is established which falls near the geometric mean of the two durations, rather than at their midpoint.

3. The experiments reported in this paper demonstrate the realtime criterion mechanism only for relatively long stimulus durations. Whether brief durations can be discriminated in the same way remains to be determined.

(Received for publication August 30, 1976; revision accepted December 27,1976 .) 